\begin{tabular}{|l|l|}
\hline 2. To: (Receiving Organization) & $\begin{array}{l}\text { 3. From: coriginating Organization) } \\
\text { Cristribution }\end{array}$ \\
\hline 5. Proj./Prog./Dept./Div.: & $\begin{array}{l}\text { 6. Design Authority/ Design Agent/Cog. } \\
\text { Engr.: }\end{array}$ \\
\hline
\end{tabular}

8. Originator Remarks:

11. Receiver Remarks:
11A. Design Baseline Document?
. Criticality and Shielding
6. Design Authority
Engr-:
S. R. Gedeon

4. Related EDT No.:

620300

7. Purchase Order No.:

NA

9. Equip./Component No.:

NA

10. System/Bldg./Facility:

12. Major Assm. Dwg - No.:

NA

13. Permit/Permit Application No.: NA

14. Required Response Date:

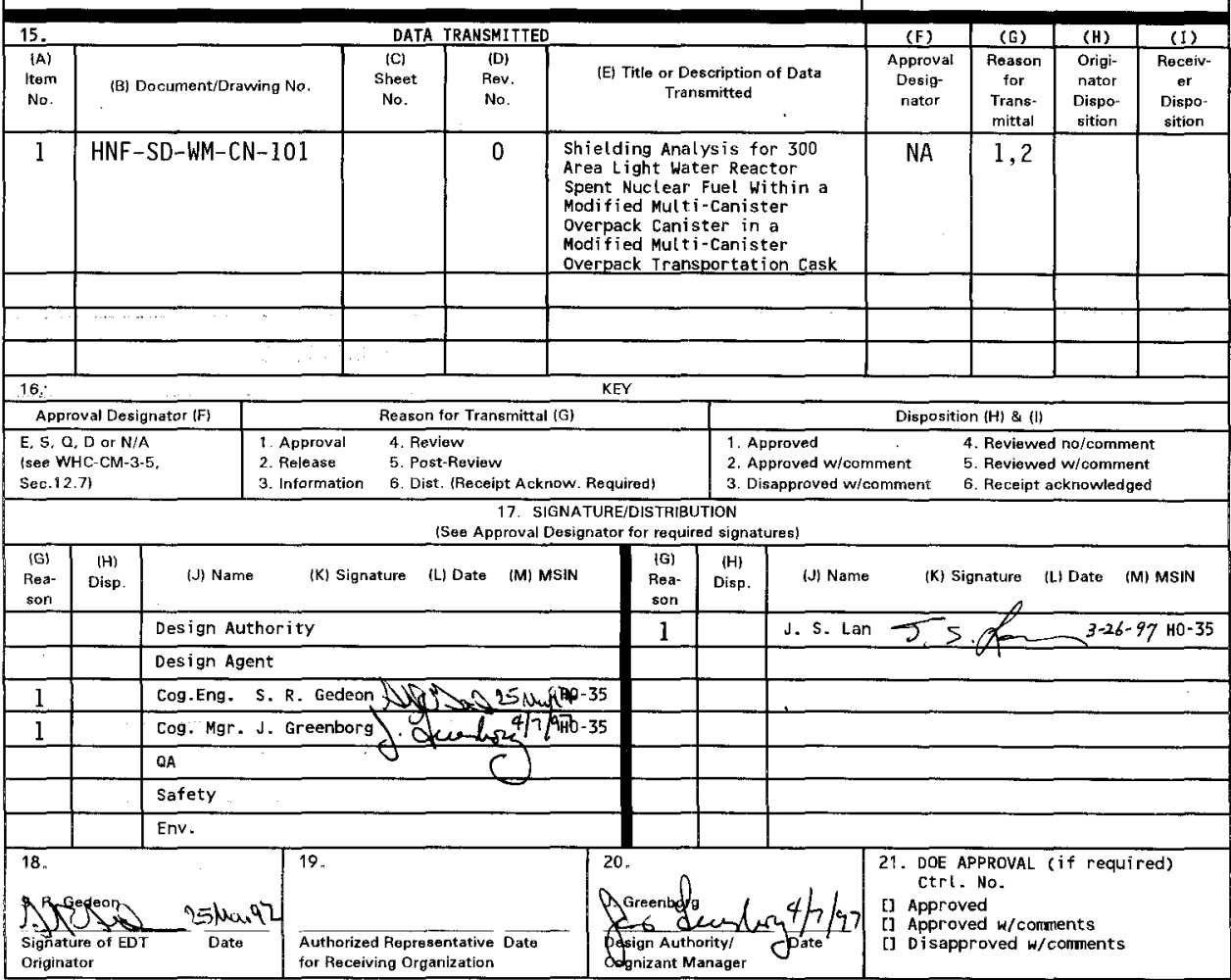




\section{Shielding Analysis for 300 Area Light Water Reactor Spent Nuclear Fuel Within a Modified Multi-Canister Overpack Canister in a Modified Multi-Canister Overpack Cask}

S. R. Gedeon

Fluor Daniel Northwest, Inc., Richland, WA 99352

U.S. Department of Energy Contract DE-AC06-96RL13200
EDT/ECN: $\quad 620300$
UC: 513
Org Code: 403
Charge Code: E58026
B\&R Code: EW7040000
Total Pages: 나 23

Key Words: Dose Rate, Overpack, LWR Fuel

Abstract: An estimate of gamma ray dose rate on the exterior of a transport cask loaded with spent LWR fuel.

IRADEMARK DISCLAIMER. Reference herein to any specific commercial product, process, or service by trade name, trademark, manufacturer, or otherwise, does not necessarily constitute or imply its endorsement, recommendation, or favoring by the United States Government or any agency thereof or its contractors or subcontractors.

Printed in the United States of America. To obtain copies of this document, contact: Document Control Services, P.O. Box 950, Mailstop H6-08, Richland WA 99352, Phone (509) 372-2420; Fax (509) 376-4989.
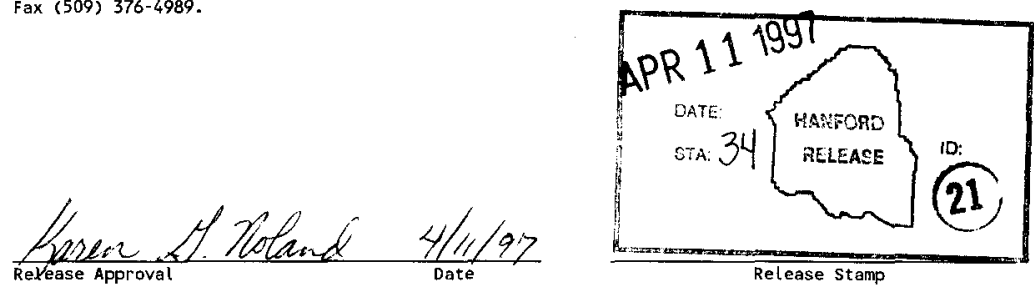

Approved for Public Release 
HNF-SD-WM-CN-101 REV 0

TABLE OF CONTENTS

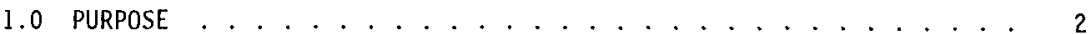

2.0 METHODOLOGY AND ASSUMPTIONS ................... 2

3.0 REFERENCES . . . . . . . . . . . . . . . . 6

APPENDIX A. ISOSHLD INPUT FILES . . . . . . . . . . . . . A-1

APPENDIX B. MCNP INPUT AND OUTPUT FILES . . . . . . . . . . . B-I

\section{LIST OF FIGURES}

Figure 1. Dose Rates vs Distance from Transport Cask Mid-Plane . . . . 3

\section{LIST OF TABLES}

Table 1. Dose Rates versus Distance at Cask Midplane (mrem/hr) . . . . 2

Table 2. Dose Rates Above the Canister Lid (12 inches above the fuel assembly) for Various Thicknesses and Lid Materials

Table 3. Dose Rates Above the Canister Lid $(6$ inches above the fuel assembly) for Various Thicknesses of Depleted Uranium $(\mathrm{rem} / \mathrm{hr})$................... . . . 4

Table 4. Dose Rate at Cask Midplane as Function of Distance, Loading and Shielding $(\mathrm{mrem} / \mathrm{hr}) \ldots . . . . . . . . .55$ 


\section{SHIELDING ANALYSIS FOR 300 AREA LIGHT WATER REACTOR SPENT NUCLEAR FUEL WITHIN A MODIFIED MULTI-CANISTER OVERPACK CANISTER IN A MODIFIED MULTI-CANISTER OVER-PACK TRANSPORTATION CASK}

\subsection{PURPOSE}

Spent light water reactor fuel is to be moved out of the 324 Building. It is anticipated that intact fuel assemblies will be loaded in a modified Multi-Canister Overpack Canister, which in turn will be placed in an Overpack Transportation Cask. An estimate of gamma ray dose rates from a transportation cask is desired.

\subsection{METHODOLOGY AND ASSUMPTIONS}

The computer code ISOSHLD (References 1, 2, and 3) was used to calculate dose rates out the side of, and along the centerline axis above, a transportation cask. Bremsstrahlung from the $B$-particle emitting radionuclides was accounted for. The fluence-to-dose conversions used here are for radiation that enters a homogenized body according to ANSI/ANS-6.1.11997.

A cylindrical source with concentric cylindrical shield geometry was used. Each fuel assembly was modelled as a cylinder of the same cross sectional area, with an active fuel length region of $366 \mathrm{~cm}$. The material composition and density of the fuel region was determined by smearing the assembly, using typical PWR dimensions from Appendix $\mathrm{H}$ of Reference 4 . The gamma source term came from Table $C .1$ of Reference 5 . The overpack canister was modelled as a cylinder of wall thickness 0.5 in $(1.27 \mathrm{~cm})$, and the transportation cask as a cylinder of wall thickness 7.31 in $(18.56 \mathrm{~cm})$. Each cask is envisioned as holding three fuel assemblies. Two geometries were modelled: one representing the assembly closest to the cask wall, the other representing one of the two (symmetrically placed) assemblies behind the first. The results given below in Figure 1 and Table 1 are the sum of the first case and twice the second.

Table 1. Dose Rates versus Distance at Cask Midplane (mrem/hr)

\begin{tabular}{|c|c|}
\hline Distance & Dose Rate \\
\hline \hline Contact (2 inch) & 650 \\
\hline 1 meter & 194 \\
\hline 6 meter & 25 \\
\hline
\end{tabular}




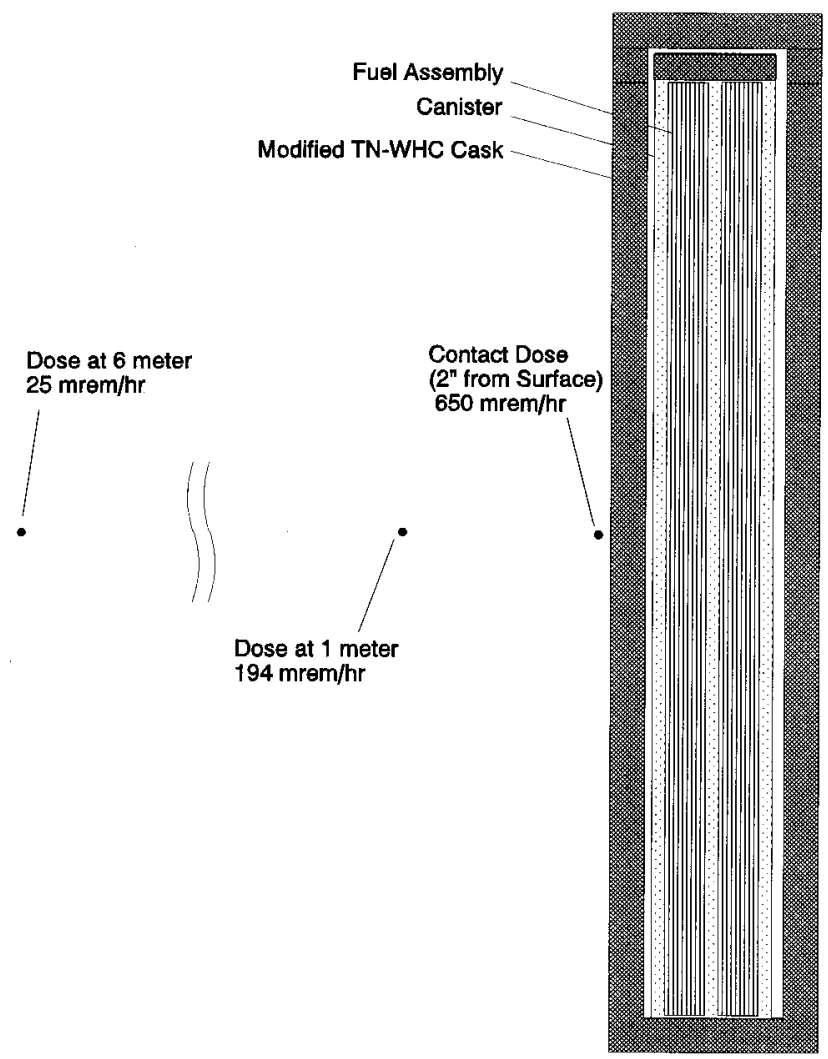

Figure 1 Dose Rate vs Distance From Transport Cask Mid-Plane 
To determine dose rates above a cask, the ISOSHLD geometry of a cylindrical source region viewed from on end, with slab shields, was used. The dose point was chosen to be 12 inches $(30.5 \mathrm{~cm})$ above the fuel assembly. The source region was given the same cross sectional area as three rectangular fuel assemblies. Shields, representing cask lids, of various thickness and material, were analyzed. Results are given in Tables 2 and 3.

Table 2. Dose Rates Above the Canister Lid (12 inches above the fuel assembly) for Various Thicknesses and Lid Materials (rem/hr)

\begin{tabular}{||l|c|c|c|c||}
\hline \hline Lid Thickness & Steel & Lead & $\begin{array}{c}\text { Depleted } \\
\text { Uranium }\end{array}$ & Tungsten \\
\hline \hline 1 inch & 640 & 91 & 15 & 34 \\
\hline 2 inches & 230 & 13 & 0.7 & 2 \\
\hline 3 inches & 78 & 2.3 & 0.04 & 0.14 \\
\hline 4 inches & 27 & 0.5 & 0.002 & 0.01 \\
\hline 6 inches & 3.1 & - & & \\
\hline 8 inches & 0.4 & - & & \\
\hline
\end{tabular}

Table 3. Dose Rates Above the Canister Lid ( 6 inches above the fuel assembly) for Various Thicknesses of Depleted Uranium ( $\mathrm{rem} / \mathrm{hr}$ )

\begin{tabular}{||l|c|}
\hline \multicolumn{1}{|c|}{ Lid Thickness } & Depleted Uranium \\
\hline \hline 1 inch & 24. \\
\hline 2 inches & 1.1 \\
\hline 3 inches & 0.05 \\
\hline 4 inches & 0.0025 \\
\hline
\end{tabular}

ISOSHLD input files are given in Appendix A.

To further refine this problem, the Monte Carlo code MCNP (References 6 and 7) was used with an existing model of the transport cask and overpack cask. Two loadings were modelled: 1 fuel assembly centered in the cask; and 2 assemblies centered side by side. For the 2 assembly case, two additional shielding geometries were analyzed: a cylindrical insert, with wall thickness of either 1 inch or 2 inches, was added. [Note: the nominal 2 inch shield was modelled with a wall thickness of $5.00 \mathrm{~cm}$, as a $5.08 \mathrm{~cm}$ thick insert does not fit.] The insert itself was modelled as either stainless steel or depleted uranium. Results are given in Table 4. 
Table 4. Dose Rate at Cask Midplane as Function of Distance, Loading and Shielding (mrem/hr)

\begin{tabular}{|c|c|c|c|c|c|c|}
\hline & 1 asbly & 2 asbly & $\begin{array}{ll}2 & \text { asbly } \\
1^{\prime \prime} & \text { steel } \\
\end{array}$ & $\begin{array}{l}2 \text { asbiy } \\
2 " \text { steel } \\
\end{array}$ & $\begin{array}{l}2 \text { asbly } \\
1 " \mathrm{DU}\end{array}$ & $\begin{array}{l}2 \text { asbly } \\
2 " \mathrm{DU} \\
\end{array}$ \\
\hline $\begin{array}{l}\text { Contact } \\
\text { (ave) }\end{array}$ & $\begin{array}{l}346 \\
(2.6)\end{array}$ & $\begin{array}{l}483 \\
(4.5)\end{array}$ & $\begin{array}{l}145 \\
(6.2)\end{array}$ & $\begin{array}{l}49.6 \\
(8.2)\end{array}$ & $\begin{array}{l}19 \\
(10)\end{array}$ & $0.3(42)$ \\
\hline $\begin{array}{l}\text { Contact } \\
\text { (x-axis) }\end{array}$ & & 501 (11) & $\begin{array}{l}187 \\
(14)\end{array}$ & 67 (27) & $15(17)$ & 1. (63) \\
\hline $\begin{array}{l}\text { Contact } \\
(\mathrm{y} \text {-axis) }\end{array}$ & & 485 (14) & $162(16)$ & $55(19)$ & $19(22)$ & 0.3 (25) \\
\hline $\begin{array}{l}1 \text { meter } \\
\text { (ave) }\end{array}$ & $\begin{array}{l}114 \\
(1.8)\end{array}$ & $\begin{array}{l}154 \\
(3.4)\end{array}$ & $47(4.7)$ & $18(6.6)$ & $\begin{array}{l}6.2 \\
(8.3) \\
\end{array}$ & 0.7 (21) \\
\hline $\begin{array}{l}2 \text { meter } \\
\text { (ave) }\end{array}$ & $60(1.9)$ & $80(3.5)$ & $26(5.0)$ & $10(7.9)$ & $\begin{array}{l}4.2 \\
(7.9)\end{array}$ & $0.9(14)$ \\
\hline $\begin{array}{l}3 \text { meter } \\
\text { (ave) }\end{array}$ & $12(2.6)$ & $17(4.7)$ & 6. $(6.3)$ & $\begin{array}{l}2 . \\
(9.4)\end{array}$ & $\begin{array}{l}1.2 \\
(10) \\
\end{array}$ & 0.4 (13) \\
\hline
\end{tabular}

Notes: 1 . Results are in $\mathrm{mrem} / \mathrm{hr}$.

2. Contact dose is calculated 2 inches from the surface.

3. The "average" dose rate is an azimuthal average at cask midplane.

The number is parentheses is the MCNP-calculated per cent uncertainty in the stated result. It should be noted that, while uncertainties of less than 10 per cent indicate generally reliable answers, uncertainties in the range of 10 to 20 percent indicate questionable results, 20 to 50 per cent indicate order of magnitude results, and results with greater than 50 per cent uncertainty should be discounted completely.

Appendix B contains a sample MCNP input file and file listing. 
HNF-SD-WM-CN-101 REV 0

\subsection{REFERENCES}

1. ISOSHLD--A Computer Code for General Purpose Isotope Shielding Analysis, BNWL-236, R. L. Enge1, J. Greenborg, M. M. Hendrickson, dated June 1966.

2. ISOSHLD-II: Code Revision to Include Calculation of Dose Rate from Shielded Bremsstrahlung Sources, G. L. Simmons, J. J. Regimbal, J. Greenborg, E. L. Ke11y, Jr., H. H. van Tuyl, dated March 1967.

3. Rittmann, P. D., CCC-636, ISO-PC Ver. 2.1, WHC-SD-WM-UM-030, Radiation Safety Information Computational Center, Oak Ridge, TN, 1996.

4. Duderstadt, J.J. \& L.J. Hamilton, Nuclear Reactor Analysis, Wiley \& Sons, New York, New York, 1976.

5. Jenquin, U. P., 1996, Inventory of LWR Spent Nuclear Fuel in the 324 Building, PNNL-11273, dated September 1996.

6. Carter, L. L., 1995, Certification of MCNP Version 4A for WHC Computer Platforms, WHC-SD-MP-SWD-30001, Rev.7, ECN 605701, Westinghouse Hanford Company, Richland, Washington, dated January 1995.

7. Breismeister, J. F., Editor, 1993, MCNP - A General Monte Carlo NParticle Transport Code, Version 4A, Los Alamos National Laboratory report LA-12625, Los Alamos, New Mexico. 
HNF-SD-WM-CN-101 REV 0

\section{APPENDIX A. ISOSHLD INPUT FILES}

02 MCO cask with LWR fuel

Case 1: Cask side, near fuel

\&INPUT NEXT $=1$, ISPEC $=3$, OPTION=0, ICONC $=0$, 1GEOM=7, DUNIT $=6$, $X=40.683, Y=183.00$, SLTH $=366.00, T(1)=12.181$

$T(2)=2.07, T(3)=1.27, T(4)=1.52, T(5)=18.562$

NPSI $=51$, NT́HETA $=51$, DÉLR $=1.0$, NSHLD $=5, J B U F=5$,

WEIGHT $(496)=9.20 \mathrm{E}+2$, WEIGHT $(499)=6.37 \mathrm{E}-0$,

WEIGHT $(498)=6.40 E+0$, WEIGHT $(505)=6.82 E+0$,

WEIGHT $(336)=2.70 \mathrm{E}+4$, WEIGHT $(206)=1.05 \mathrm{E}+1$,

WEIGHT $(504)=5.27 E+0$, WEIGHT $(506)=4.68 E+0$,

WEIGHT $(500)=4.26 E+2$, WEIGHT $(472)=7.88 E+2$,

WEIGHT $(319)=3.07 E+2$, WEIGHT $(335)=2.85 E+4$.

WEIGHT $(415)=1.20 E+3$, WEIGHT $(418)=2.97 E+2$

WEIGHT $(290)=1.26 \mathrm{E}-2$, WEIGHT $(55)=1.31 \mathrm{E}+3$,

WEIGHT (502) $=1.34 \mathrm{E}-1$, WEIGHT (503) $=6.82 \mathrm{E}+0$,

WEIGHT (388) $=8.17 E+2, W E I G H T(492)=1.04 E+3$,

WEIGHT(493) $=1.25 E+2$, WEIGHT (494) $=2.12 E+2$,

WEI GHT (495) $=2.38 \mathrm{E}+4$, WEIGHT (497) $=6.84 \mathrm{E}-1$,

WEIGHT $(172)=4.36 E+0$, WEIGHT $(170)=4.34 E+0$,

WEIGHT (269) $=1.05 E+2$, WEI GHT (403) $=1.23 E+2$,

WEIGHT $(B 2)=1.98 E+4$, WEIGHT $(141)=5.21 E+0$,

WEIGHT $(270)=2.56 E+1$, WEIGHT $(520)=4.72 E-1$,

WEIGHT $(476)=6.91 \mathrm{E}-3$, WEIGHT $(491)=5.83 \mathrm{E}-1$,

WEIGHI (526) $=1.27 \mathrm{E}-1$, WEIGHT $(84)=1.98 \mathrm{E}+4$

\&

$\begin{array}{lrrrrrr}\text { air } & 3 & 0.0 & 0.00122 & 0.0 & 0.00122 & 0.0 \\ \text { u: f } & 15 & 2.652 & 0.0 & 0.0 & 0.0 & 0.0 \\ \text { o: f } & 23 & 0.3565 & 0.0 & 0.0 & 0.0 & 0.0 \\ z r=f & 11 & 0.2993 & 0.0 & 0.0 & 0.0 & 0.0 \\ \mathrm{Fe} & 09 & 0.0 & 0.0 & 7.8 & 0.0 & 7.8\end{array}$

dose rate 1 meter from side

\&INPUT NEXT $=4, X=135.603 \&$

dose rate 6 meter from side

\&INPUT NEXT $=4, X=635.603 \&$

end

\&INPUT NEXT $=6$ \&

0 2 MCO cask with LWR fuel

Case 2: Cask side, far fuel

\& INPUT NEXT $=1$, I SPEC $=3$, OPT ION=0, [ $\mathrm{CONC}=0$, I $\mathrm{GEOM}=7$, DUNIT $=6$, $X=63.406, Y=183.00$, SLTH $T$ = 366.00, $T(1)=12.181$

$T(2)=22.72, T(3)=1.27, T(4)=3.59, T(5)=18.562$

NPSI $=51$, NTHETA $=51, D E L R=1.0, N S H L D=5$, JBUF $=5$,

WEIGHT $(496)=9.20 \mathrm{E}+2$, WEI GHT $(499)=6.37 \mathrm{E}-0$,

WE I GHT (498) $=6.40 \mathrm{E}+0$, WE I GHT $(505)=6.82 \mathrm{E}+0$,

WEIGHT $(336)=2.70 E+4$, WEIGHT $(206)=1.05 E+1$,

WEI GHT $(504)=5.27 \mathrm{E}+0$, WEIGHT $(506)=4.68 \mathrm{E}+0$,

WEIGHT $(500)=4.26 \mathrm{E}+2$, WEIGHT $(472)=7.88 \mathrm{E}+2$

WEIGHT (319) $=3.07 E+2$, WEIGHT $(335)=2.85 E+4$,

WE I GHT (415) $=1.20 E+3$, WEIGHT $(418)=2.97 \mathrm{E}+2$

WEIGHT $(290)=1.26 \mathrm{E}-2$, WEIGHT $(55)=1.31 \mathrm{E}+3$,

WEIGHT $(502)=1.34 \mathrm{E}-1$, WEIGHT $(503)=6.82 \mathrm{E}+0$,

WEIGHT (388) $=8.17 E+2$, WEIGHT $(492)=1.04 E+3$

WE $I G H T(493)=1.25 E+2$, WEIGHT $(494)=2.12 E+2$,

WE I GHT (495) $=2.38 \mathrm{E}+4$, WEI IGHT $(497)=6.84 \mathrm{E}-1$,

WEI GHT $(172)=4.36 \mathrm{E}+0$, WEIGHT $(170)=4.34 \mathrm{E}+0$

WEIGHT (269) $=1.05 E+2$, WEIGHT $(403)=1.23 E+2$,

WEIGHT $(82)=1.98 E+4$, WEIGHT $(141)=5.21 \mathrm{E}+0$,

WEIGHT $(270)=2.56 \mathrm{E}+1$, WE I GHT $(520)=4.72 \mathrm{E}-1$,

$\operatorname{WEIGHT}(476)=6.91 \mathrm{E}-3$, WEIGHT $(491)=5.83 \mathrm{E}-1$,

WEIGHT (526) $=1.27 \mathrm{E}-1$, WE I GHT $(84)=1.98 \mathrm{E}+4$

\&

$\begin{array}{lllllll}\text { air } & 3 & 0.0 & 0.00000 & 0.0 & 0.00122 & 0.0\end{array}$ 


\section{HNF-SD-WM-CN-101. REV 0}

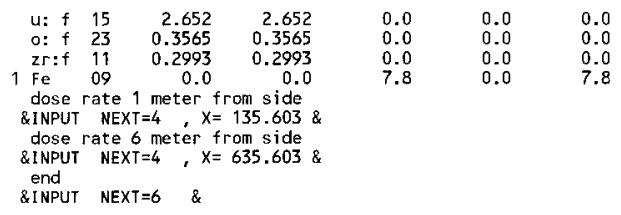

02300 Area LWR fuel

Case 3a: top, $1 \mathrm{ft}$ above fuel assembly, steel plug--1 inch \&INPUT NEXT $=1$, I SPEC $=3$, OPTION=0, I $C O N C=0$, I GEOM=9, DUNIT $=6$, SFACT $=3.0, X=416.426, \quad S L T H=21.098, T(1)=366.00$,

$T(2)=19.946, T(3)=2.54$

NPSI $=51$, NTHETA $=51, \mathrm{DELR}=1.0, \mathrm{NSHLD}=3, J B U F=3$,

WEIGHT (496) $=9.20 \mathrm{E}+2$, WEIGHT $(499)=6.37 \mathrm{E}-0$,

WEI IHHT (498) $=6.40 E+0$, WEIGHT $(505)=6.82 E+0$,

WEIGHT (336) $=2.70 E+4$, WEIGHT $(206)=1.05 E+1$,

WEIGHT (504) $=5.27 \mathrm{E}+0$, WEIGHT $(506)=4.68 \mathrm{E}+0$,

WEIGHT $(500)=4.26 \mathrm{E}+2$, WEIGHT $(472)=7.88 \mathrm{E}+2$

WEI GHT $(319)=3.07 E+2$, WEIGHT $(335)=2.85 \varepsilon+4$,

WEIGHT $(415)=1.20 \mathrm{E}+3$, WEIGHT $(418)=2.97 \mathrm{E}+2$,

WEIGHT $(290)=1.26 \mathrm{E}-2$, WEIGHT( 55$)=1.31 \mathrm{E}+3$,

WEIGHT $(502)=1.34 \mathrm{E}-1$, WEIGHT $(503)=6.82 \mathrm{E}+0$,

WEIGHT (388) $=8.17 E+2$, WEIGHT(492) $=1.04 E+3$,

WEIGHT (493) $=1.25 E+2$, WEIGHT $(494)=2.12 E+2$,

WEIGHT $(495)=2.38 E+4$, WEIGHT $(497)=6.84 E-1$,

WEIGHT $(172)=4.36 \mathrm{E}+0$, WEIGHT $(170)=4.34 \mathrm{E}+0$

WEIGHT $(269)=1.05 E+2$, WEIGHT $(403)=1.23 E+2$,

WEIGHT $(82)=1.98 E+4$, WEIGHT (141) $=5.21 E+0$,

$\operatorname{WEIGHT}(270)=2.56 E+1, \operatorname{WEIGHT}(520)=4.72 E-1$,

WEIGHT $(476)=6.91 \mathrm{E}-3$, WEIGHT $(491)=5.83 \mathrm{E}-1$,

WEIGHT $(526)=1.27 \mathrm{E}-1$, WEIGHT $(84)=1.98 \mathrm{E}+4$

\&

$\begin{array}{lrrrr}\mathrm{u}: \mathrm{f} & 15 & 2.652 & 0.0 & 0.0 \\ 0: f & 23 & 0.3565 & 0.0 & 0.0 \\ \mathrm{zr}: \mathrm{f} & 11 & 0.2993 & 0.2993 & 0.0 \\ \mathrm{Fe} & 09 & 0.0 & 0.0 & 7.8\end{array}$

Case 3b: top, $1 \mathrm{ft}$ above fuel assembly, steel plug--2 inch \&INPUT NEXT $=4, T(3)=5.08 \&$

Case $3 \mathrm{c}$ : top, $1 \mathrm{ft}$ above fuel assembly, steel plug- -3 inch \&INPUT NEXT $=4, T(3)=7.62 \&$

Case 3d: top, $1^{\prime} \mathrm{ft}$ above fuel assembly, steel plug- 4 inch \&INPUT NEXT $=4, \mathbf{I}(3)=10.16$ \&

end

\&INPUT NEXT $=6$ \&

02300 Area LWR Fuel

Case 3a: top, $1 \mathrm{ft}$ above fuel assembly, steel plug -6 inch \&INPUT NEXT $=1$, ISPEC $=3$, OPIION=0, I CONC $=0$, I GEOM=9, DUNIT=6, SFACT $=3.0, X=416.426, \quad \mathrm{SLTH}=21.098, \mathrm{~T}(1)=366.00$,

$T(2)=19.946, T(3)=15.24$,

NPSI $=51$, NTHETA $=51, D E L R=1.0, N S H L D=3, J B U F=3$,

WEIGHT (496) $=9.20 \mathrm{E}+2$, WEIGHT (499) $=6.37 \mathrm{E}-0$,

WEIGHT (498) $=6.40 E+0$, WEIGHT $(505)=6.82 E+0$,

WEIGHT (336) $=2.70 E+4 ;$, WEIGHT $(206)=1.05 E+1$,

WEIGHT $(504)=5.27 \mathrm{E}+0, \mathrm{WEIGHT}(506)=4.68 \mathrm{E}+0$,

WEIGHT $(500)=4.26 \mathrm{E}+2$, WEIGHT $(472)=7.88 \mathrm{E}+2$,

WEIGHT $(319)=3.07 E+2$, WEIGHT $(335)=2.85 E+4$,

WEIGHT $(415)=1.20 E+3$, WEIGHT $(418)=2.97 E+2$,

WEIGHT $(290)=1.26 \mathrm{E}-2$, WEIGHT $(55)=1.31 \mathrm{E}+3$,

WEIGHT (502) $=1.34 \mathrm{E}-1$, WEIGHT $(503)=6.82 \mathrm{E}+0$

WEIGHT (388) $=8.17 \mathrm{E}+2$, WEIGHT (492) $=1.04 \mathrm{E}+3$

WEIGHT (493) $=1.25 E+2$, WEIGHT (494) $=2.12 E+2$,

WEIGHT (495) $=2.38 E+4$, WEIGHT $(497)=6.84 E-1$, 
WEIGHT $(172)=4.36 \mathrm{E}+0$, WEIGHT $(170)=4.34 \mathrm{E}+0$, WEIGHT $(269)=1.05 \mathrm{E}+2$, WEIGHT $(403)=1.23 \mathrm{E}+2$, WEIGHT $(82)=1.98 E+4$, WEIGHT $(141)=5.21 \mathrm{E}+0$, WEI GHT $(270)=2.56 \mathrm{E}+1$, WEIGHT $(520)=4.72 \mathrm{E}-1$, WEIGHT $(476)=6.91 \mathrm{E}-3$, WEIGHT $(491)=5.83 \mathrm{E}-1$, WEIGHT $(526)=1.27 E-1$, WEIGHT $(84)=1.98 \mathrm{E}+4$ \&

$\begin{array}{lrrrr}\text { u: } f & 15 & 2.652 & 0.0 & 0.0 \\ 0: f & 23 & 0.3565 & 0.0 & 0.0 \\ \text { zr:f } & 1 \uparrow & 0.2993 & 0.2993 & 0.0 \\ \mathrm{Fe} & 09 & 0.0 & 0.0 & 7.8\end{array}$

Case 3b: top, $1 \mathrm{ft}$ above fuel assembly, steel plug--8 inch \&INPUT NEXT $=4, T(3)=20.32$ \&

end

\&INPUT NEXT $=6$ \&

02300 Area LWR Fuel

Case 4a: top, $1 \mathrm{ft}$ above fuel assembly, lead plug- -1 inch \&INPUT NEXT $=1$, ISPEC $=3$, OPTI ION=0, I CONC $=0$, I GEON=9, DUNI $=6$, SFACT $=3.0, X=416.426, \quad S L T H=21.098, T(1)=366.00$, $T(2)=19.946, T(3)=2.54$.

NPS $1=51$, NTHETA $=51, D E L R=1.0$, NSHLD $=3, J B U F=3$, WEIGHT $(496)=9.20 \mathrm{E}+2$, WE J GHT $(499)=6.37 \mathrm{E}-0$, WEIGHT $(498)=6.40 E+0$, WE ] GHT $(505)=6.82 E+0$, WEIGHT (336) $=2.70 E+4$, WEI GHT $(206)=1.05 E+1$ WEIGHT $(504)=5.27 E+0$, WEIGHT $(506)=4.68 E+0$, WEIGHT $(500)=4.26 \mathrm{E}+2$, WEI IGHT $(472)=7.88 \mathrm{E}+2$, WEIGHT $(319)=3.07 \mathrm{E}+2$, WEIGHT $(335)=2.85 \mathrm{E}+4$, WEIGHT $(415)=1.20 E+3$, WEIGHT $(418)=2.97 \mathrm{E}+2$, WEIGHT $(290)=1.26 E-2$, WEIGHT $(55)=1.31 E+3$. WEIGHT (502) $=1.34 E-1$, WEIGHT $(503)=6.82 E+0$. WEIGHT $(388)=8.17 E+2$, WEIGHT $(492)=1.04 E+3$, $\operatorname{WEIGHT}(493)=1.25 \mathrm{E}+2, \operatorname{WEIGHT}(494)=2.12 \mathrm{E}+2$, WEIGHT(495) $=2.38 E+4$, WEIGHT (497) $=6.84 E-1$, WEIGHT $(172)=4.36 E+0$, WEIGHT $(170)=4.34 E+0$, WEIGHT (269) $=1.05 E+2$, WEIGHT $(403)=1.23 E+2$, WEIGHT $(82)=1.98 \mathrm{E}+4$, WEIGHT $(141)=5.21 \mathrm{E}+0$ WEIGHT $(270)=2.56 \mathrm{E}+1$, WEI GHT $(520)=4.72 \mathrm{E}-1$, WE IGHT (476) $=6.91 \mathrm{E}-3$, WEIGHT (491) $=5.83 E-1$, WEIGHT $(526)=1.27 \mathrm{E}-1$, WEIGHT $(84)=1.98 \mathrm{E}+4^{\circ}$ \&

$\begin{array}{rrrrr}\text { u: } f & 15 & 2.652 & 0.0 & 0.0 \\ \mathrm{o:}: \mathrm{f} & 23 & 0.3565 & 0.0 & 0.0 \\ \mathrm{zr}: \mathrm{f} & 11 & 0.2993 & 0.2993 & 0.0 \\ \mathrm{~Pb} & 14 & 0.0 & 0.0 & 11.35\end{array}$

Case 4b: top, $1 \mathrm{ft}$ above fuel assembly, lead plug- -2 inch \&INPUT NEXT $=4, T(3)=5.08$ \&

Case 4c: top, $1 \mathrm{ft}$ above fuel assembly, lead plug--3 inch \&INPUT NEXT $=4, T(3)=7.62 \&$

Case 4d: top, 1 ft above fuel assembly, lead plug- -4 inch \&INPUT NEXI $=4, T(3)=10.16 \&$

end

\&INPUT NEKT $=6 \quad \&$

03300 Area LWR Fuel

Case 5a: top, $1 \mathrm{ft}$ above fuel assembly, DU plug--1 inch \&INPUT NEXT $=1$; I $S P E C=3$, OPT ION=0, I CONC $=0$, I GEOM=9, DUNI $T=6$, SFACT $=3.0, X=416.426, \quad S L T H=21.098, T(1)=366.00$, $T(2)=19.946, T(3)=2.54$.

NPSI $=51$, NTHETA $=51$, DELR $=1.0$, NSHLD $=3, J B U F=3$

WE I GHT $(496)=9.20 E+2$, WE I GHT $(499)=6.37 E-0$, WEIGHT $(498)=6.40 E+0$, WEIGHT $(505)=6.82 E+0$, WE IGHT (336) $=2.70 \mathrm{E}+4$, WEIGHT $(206)=1.05 \mathrm{E}+1$; WEIGHT (504) $=5.27 \mathrm{E}+0$, WEIGHT (506) $=4.68 \mathrm{E}+0$, WEIGHT $(500)=4.26 E+2$, WEIGHT $(472)=7.88 E+2$, WEIGHT $(319)=3.07 E+2$, WEIGHT $(335)=2.85 E+4$, 


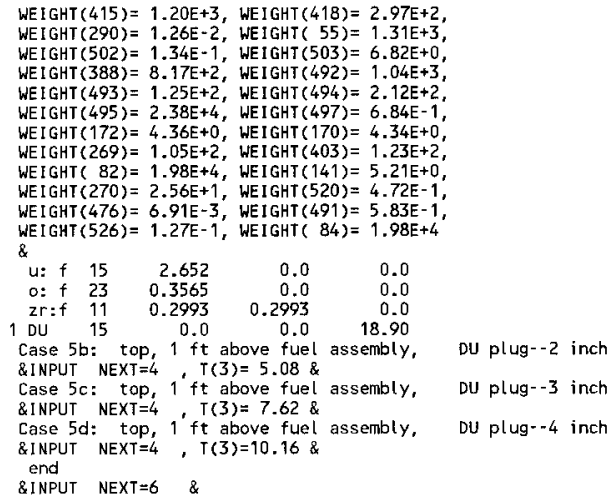




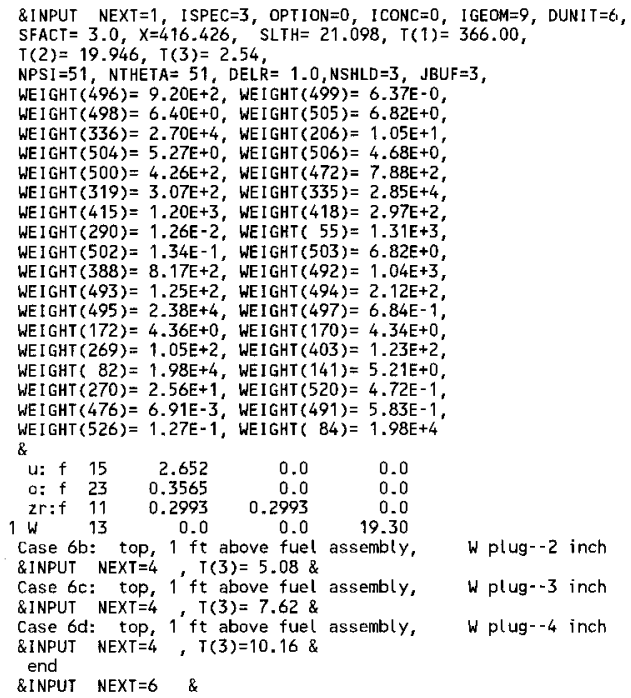




\section{APPENDIX B. MCNP INPUT AND OUTPUT FILES}

\section{INPUT/OUTPUT FILES}

The MCNP input and output files can be found in the directory: /home/w80502/projects/1wr324.

Iwrl, llout, llout2: Input and output files for the 1 fuel assembly load, no insert shield case.

1wr2, 12out, 12out2: 2 fuel assembly load, no insert shield.

1wr3, 13out, 13out2: 2 fuel assembly load, 1 inch steel insert shield.

1wr4, 140ut, 140ut2: 2 fuel assembly load, 2 inch steel insert shield.

1wr5, 15out, 15out2: 2 fuel assembly load, 1 inch DU insert shield.

1wr6, 16out, 16out2: 2 fuel assembly 1oad, 2 inch DU insert shield,

Sample input file: $1 \mathrm{wr} 3$

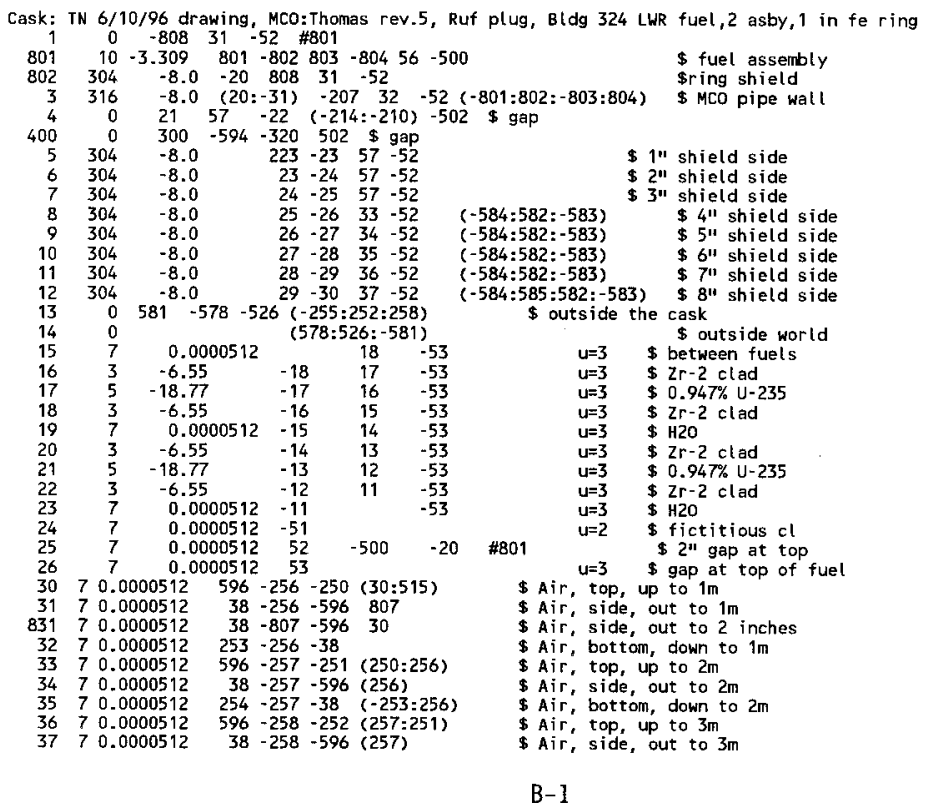




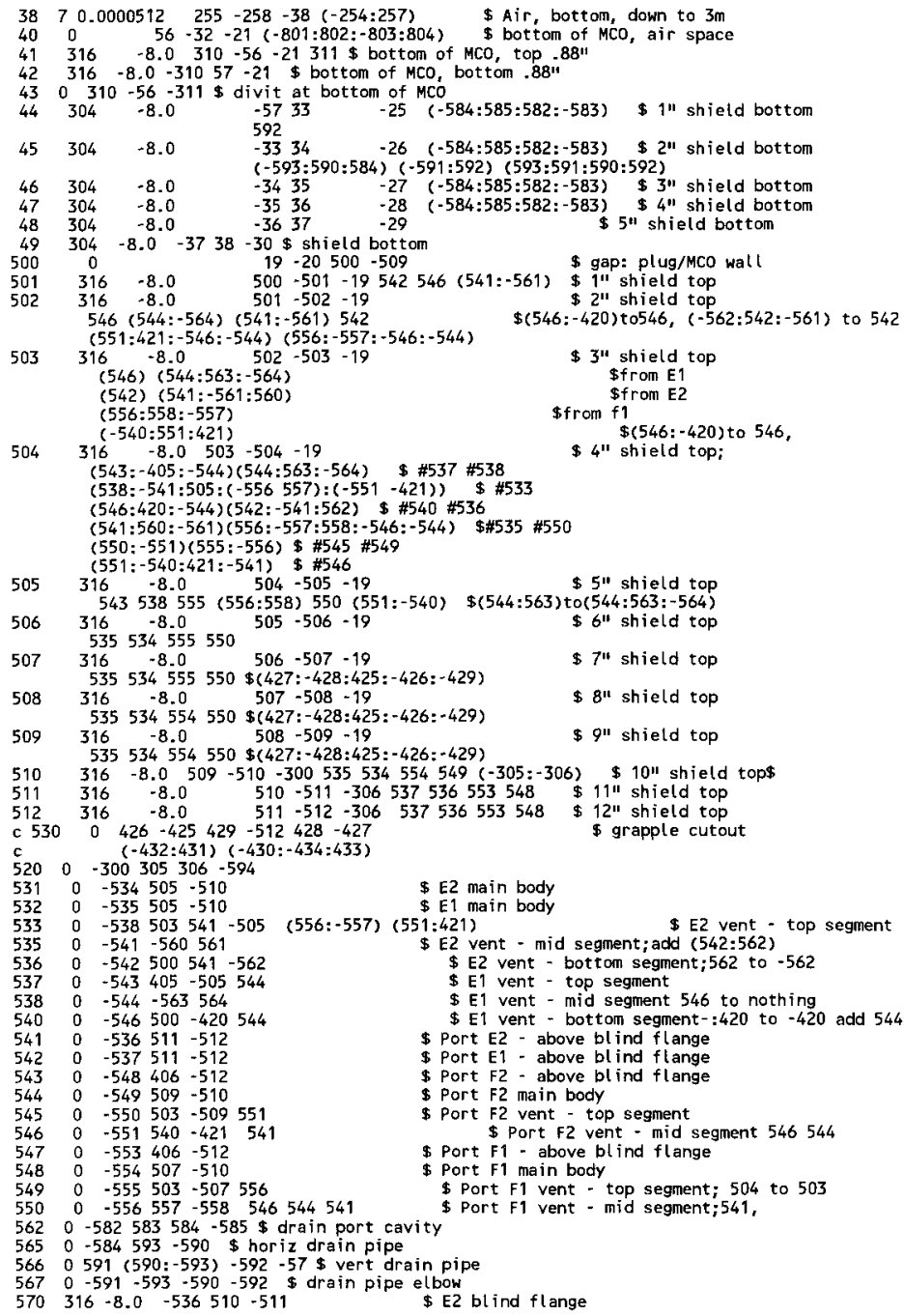




\section{HNF-SD-WM-CN-101 REV 0}

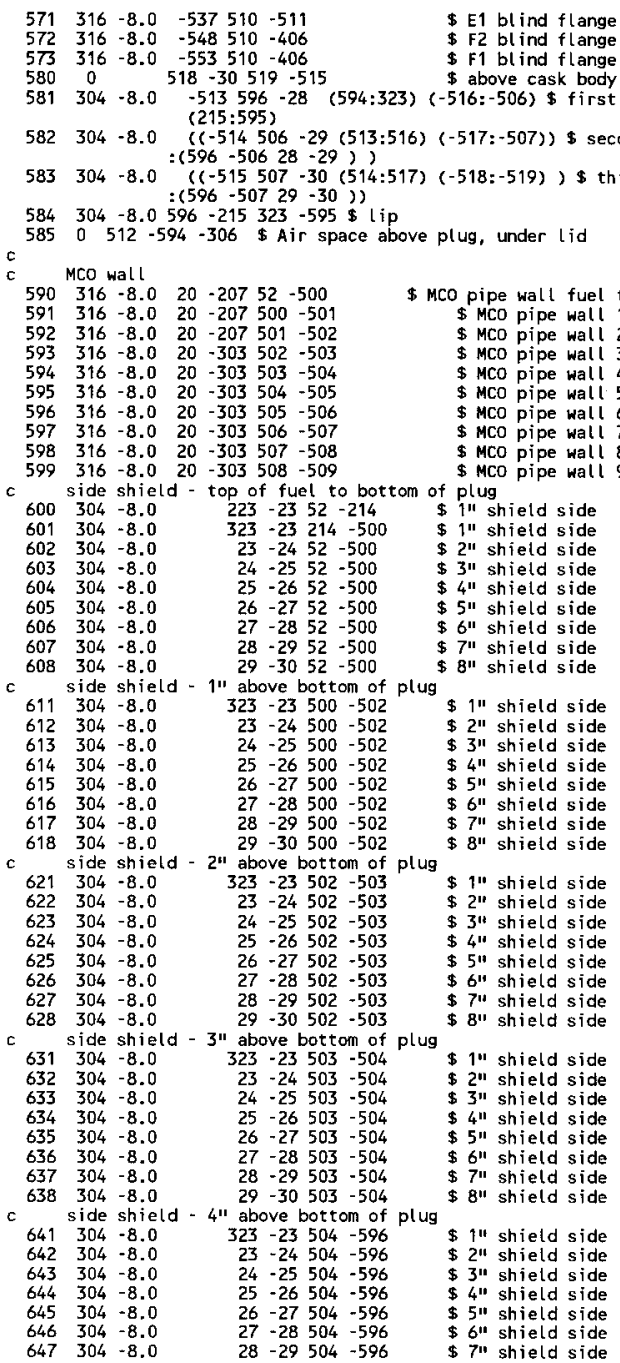


HNF-SD-WM-CN-101 REV 0

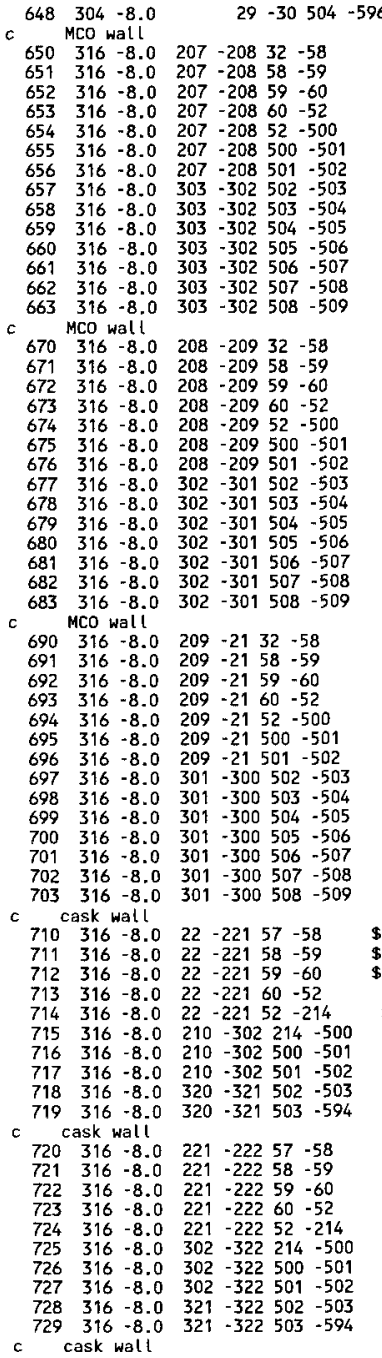

\$" shield side

$\$$ MCo pipe wall from bottom of MCO $\$$ MCO pipe wall from bottom of MCO $\$$ MCO pipe wall trom bottom of MCO $\$$ MCO pipe wall from bottom of MCO

\$ MCO pipe wall fuel to plug bottom \$ MCO pipe wall 1"+plug bottom \$ MCO pipe wall 2"+plug bottom \$ MCO pipe wall $3^{4+p l u g ~ b o t t o m ~}$ \$ MCO pipe wall 4"+plug bottom \$ MCO pipe wall 5"+plug bottom \$ MCO pipe wall $6^{11+p l u g}$ bottom \$ MCO pipe wall $7^{11+p l u g}$ bottom $\$$ MCO pipe wall 8"+plug bottom \$ MCO pipe wall 9"+plug bottom

$\$ M C O$ pipe wall from bottom of MCO $\$ M C O$ pipe wall from bottom of MCO $\$$ MCO pipe wall from bottom of MCO $\$$ MCO pipe wall from bottom of MCO

$\$$ MCO pipe wall fuel to plug botton $\$$ MCO pipe wall 1"+plug bottom $\$$ MCO pipe wall $2^{\prime \prime+p l u g ~ b o t t o m ~}$ \$ MCO pipe wall $3^{\prime \prime p l u g ~ b o t t o m ~}$ \$ MCO pipe wall $4^{1+p l u g}$ bottom \$ MCO pipe wall $5^{11+p l u g}$ bottom \$ MCO pipe wall $6^{\prime \prime+p l u g ~ b o t t o m ~}$ $\$$ MCO pipe wall $7^{\prime \prime}+p l u g$ bottom \$ MCO pipe wall $8^{11+p l u g}$ botton $\$$ MCO pipe wall $9^{1 "+p l u g}$ botton

$\$$ MCO pipe wall from bottom of MCO $\$$ MCO pipe wall from bottom of MCO $\$$ MCO pipe wall from bottom of MCO $\$$ MCO pipe wall from bottom of MCO

$\$$ MCO pipe wall fuel to plug bottom $\$$ MCO pipe wall 1"+plug botton \$MCO pipe wall 2"+plug bottorn $\$$ MCO pipe wall $3^{\prime \prime+p l u g}$ botton $\$$ MCO pipe wall 4"+plug botton \$ MCO pipe wall 5"+plug botton $\$$ MCO pipe wall 6"+plug bottom \$ MCO pipe wall $7^{\text {"t+plug bottom }}$ $\$$ MCO pipe wall g"+plug bottom $\$$ MCO pipe wall Q"+plug bottom

$\$$ 1" side shield from bottom of MCO \$ 1" side shield from bottom of MCO \$ 1" side shield from bottom of MCO \$1" side shield from bottom of MCO \$ "i" side shield fuel to plug bottom

\$ q" side shield fuel to plug bottom \$1" side shield 1"+plug bottom $\$ 1 "$ side shield $2^{11}+$ plug bottom \$1" side shield $3^{11+p l u g}$ bottom \$1" side shield 4"+plug bottom

$\$ 1 "$ side shield from bottom of MCO $\$$ 1" side shield from bottom of MCO $\$$ q" side shield from bottom of MCO $\$$ q" side shield from bottom of MCO $\$ 11$ side shield fuel to plug bottom $\$ 11$ side shield fuel to plug bottom \$1" side shield 1"+plug bottom \$1" side shield 2"+plug bottom $\$$ 1" side shield 3"+plug bottom $\$ 11$ side shield 4"+plug bottom 


\section{HNF-SD-WM-CN-101 REV 0}

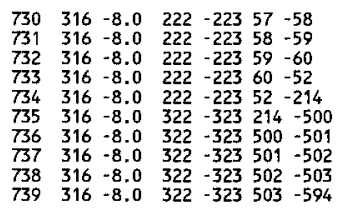

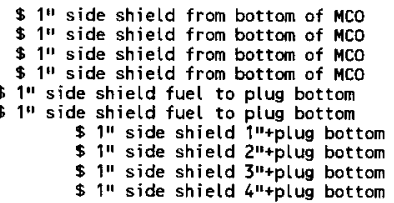

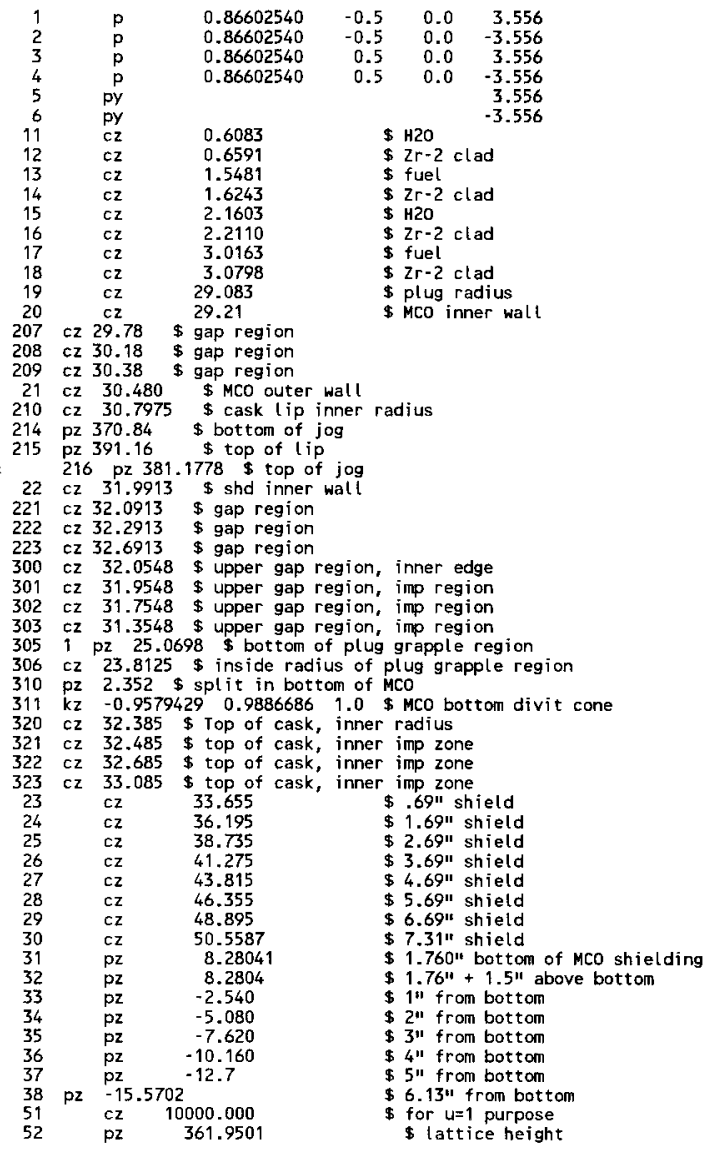




\section{HNF-SD-WM-CN-101 REV 0}

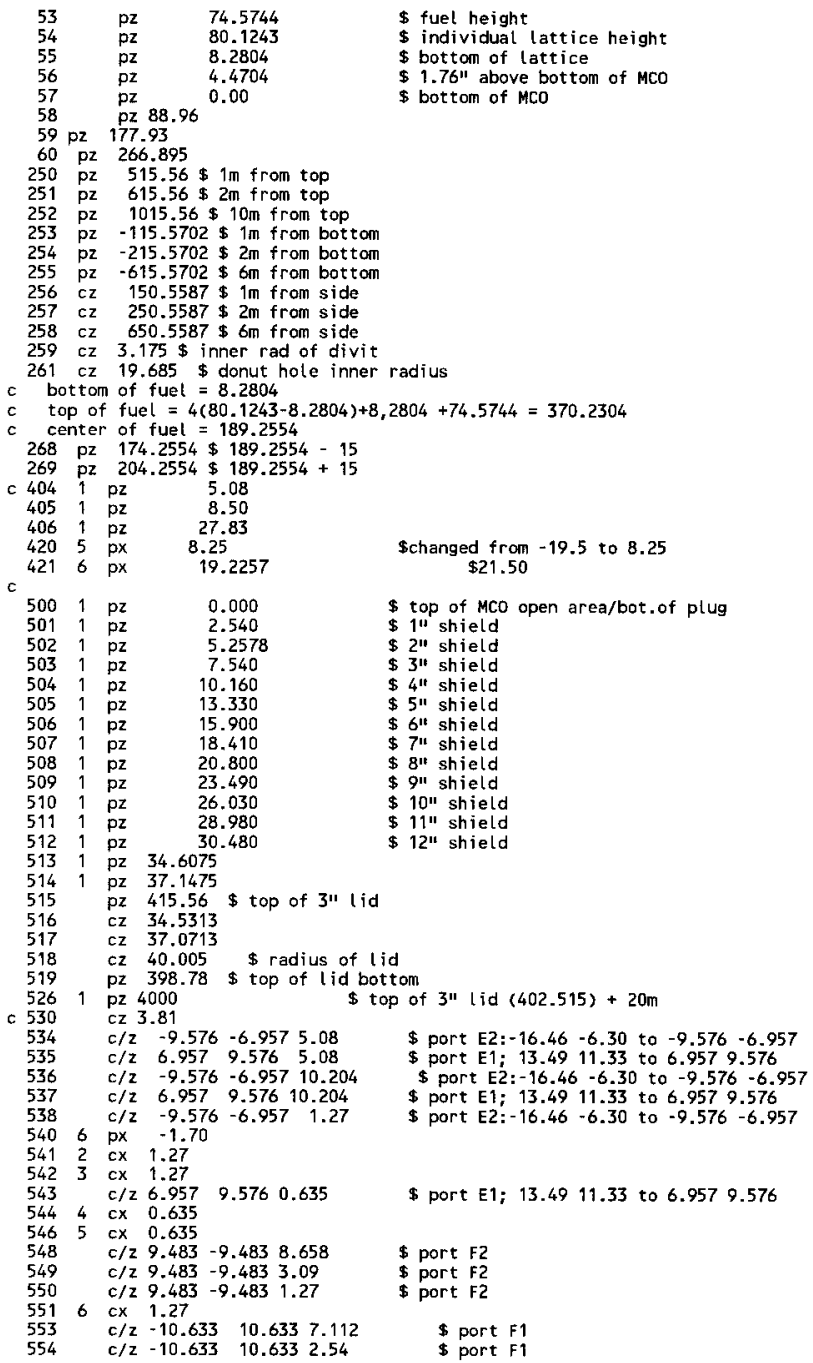




\section{HNF-SD-WM-CN-101 REV 0}

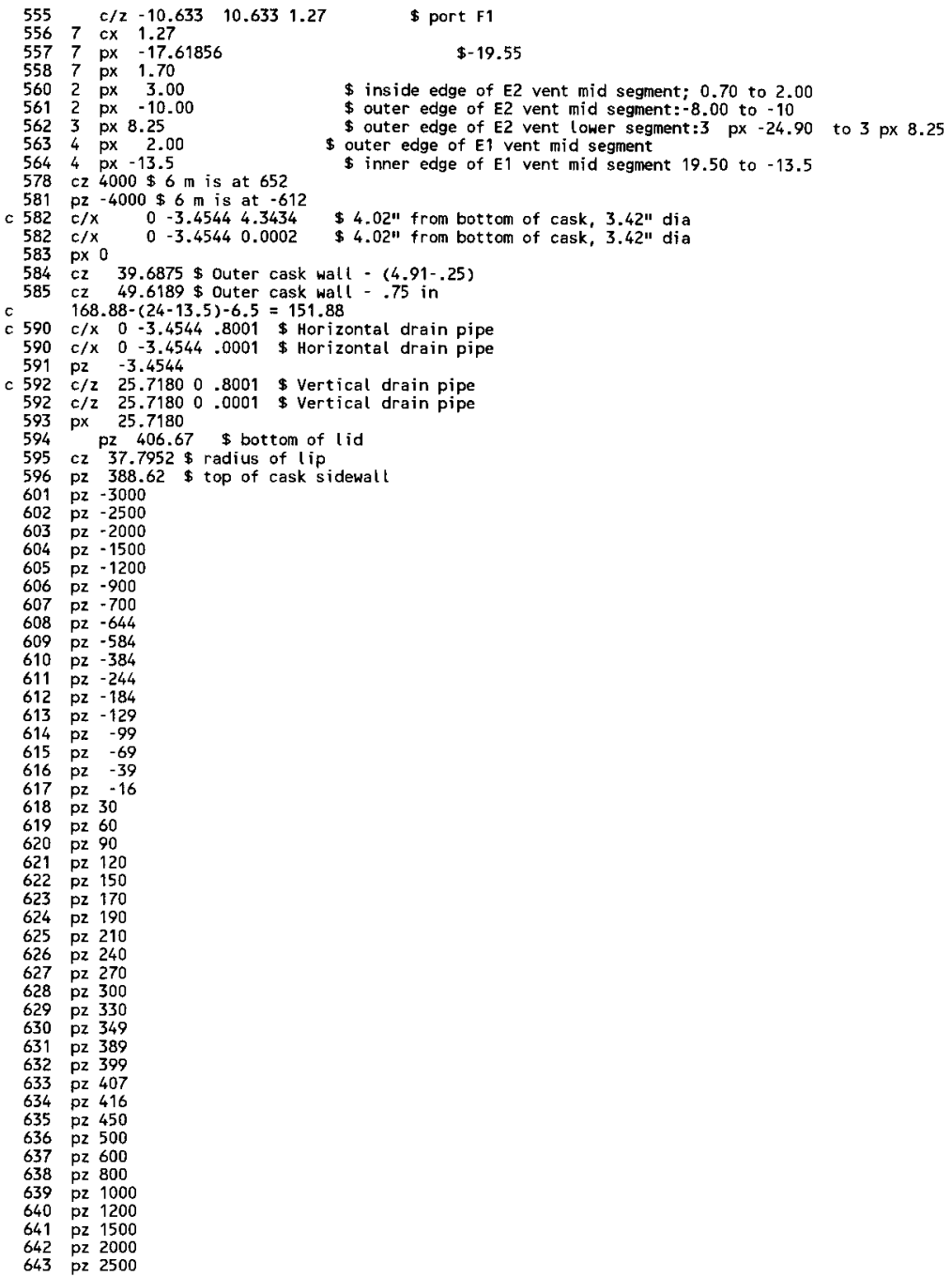




\section{HNF-SD-WM-CN-101 REV 0}

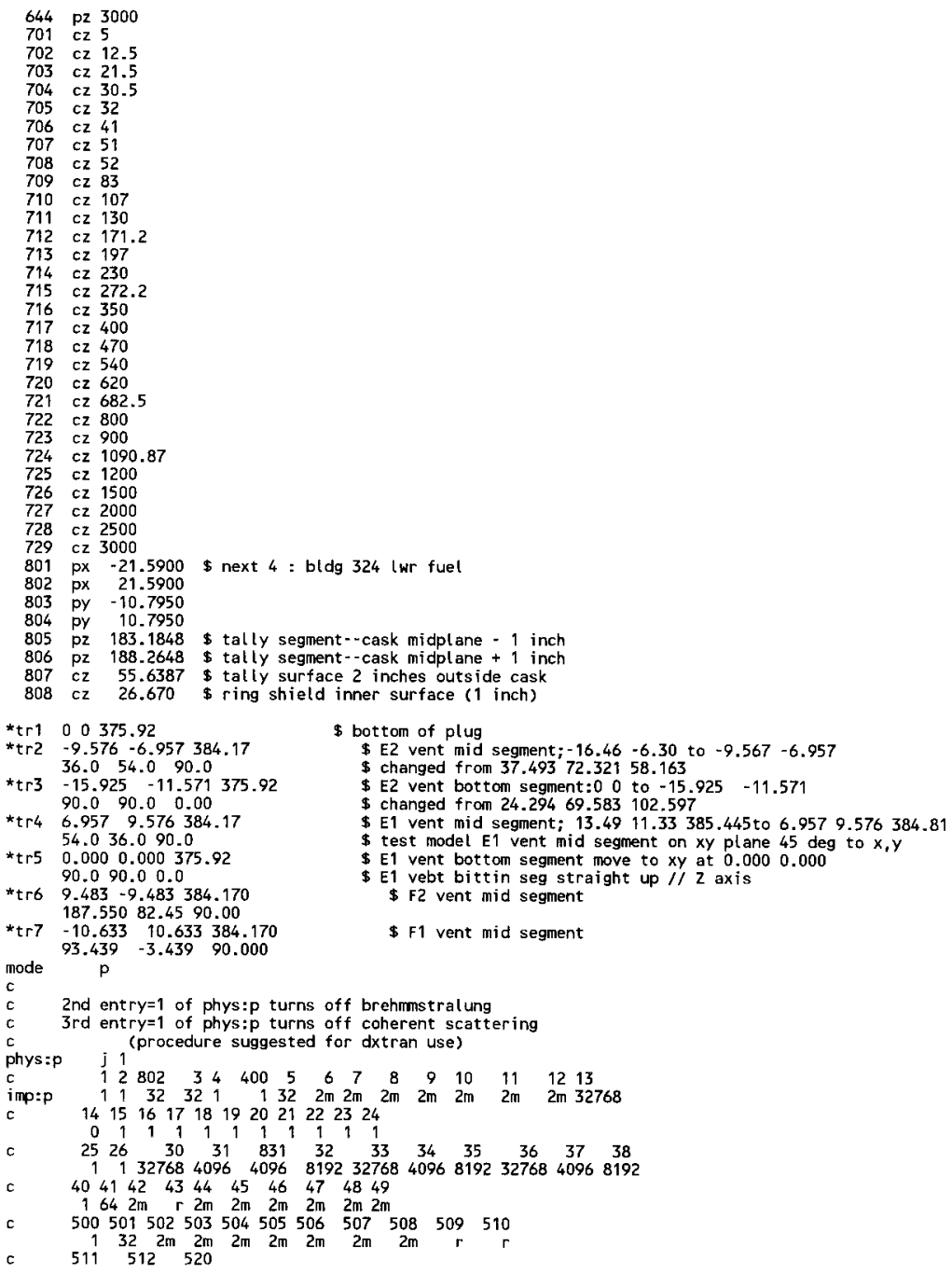




\section{HNF-SD-WM-CN-101 REV 0}

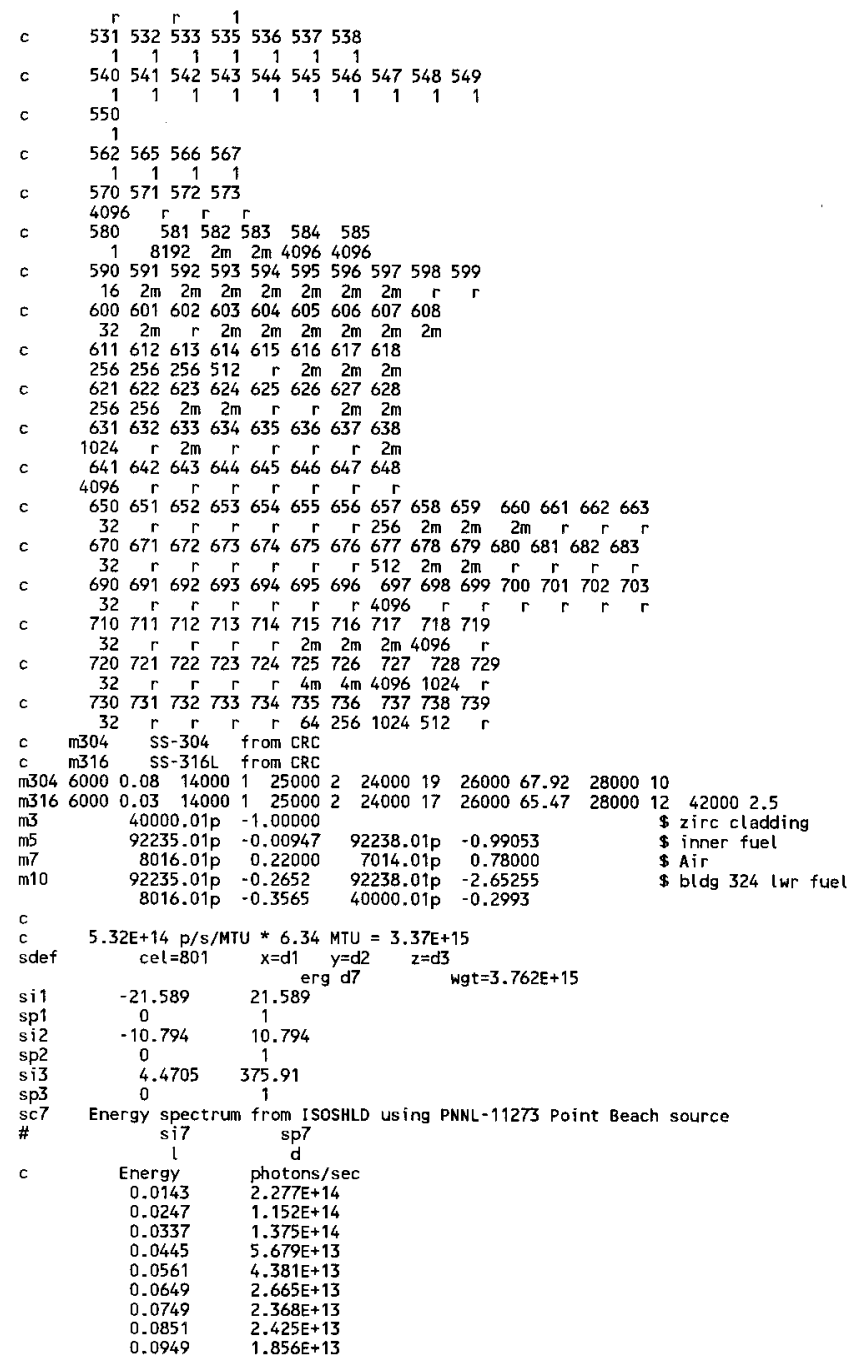




\section{HNF-SD-WM-CN-101 REV 0}

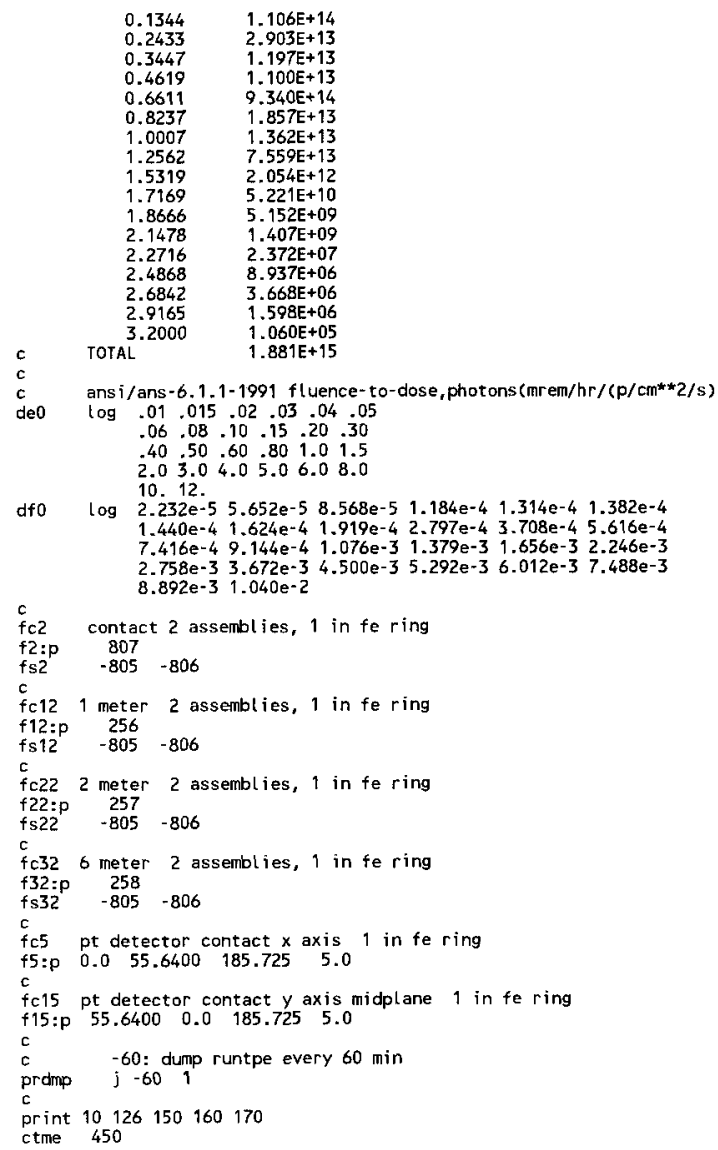




\section{CHECKLIST FOR INDEPENDENT TECHNICAL REVIEW}

NUMBER: DESH-9750369

DOCUMENT REVIEWED: Shielding Analys is for 300 Area LWR SNF Within Modified

$\operatorname{AUTHOR}(s):$ S. R. Gedeon MCO Canister in a Modified MCO Cask

I. Method(s) of Review

(x ) Input data checked for accuracy

( ) Independent calculation performed

(x) Hand calculation

( ) Alternate computer code:

( ) Comparison to experiment or previous results

() Alternate method (define)

II. Checklist (either check or enter NA if not applied)

( $x$ ) Task completely defined

( $x$ ) Activity consistent with task specification

( $x$ ) Necessary assumptions explicitly stated and supported

( $x$ ) Resources properly identified and referenced

(x) Resource documentation appropriate for this application

( $x$ ) Input data explicitly stated

( $x$ ) Input data verified to be consistent with original source

( $x$ ) Geometric model adequate representation of actual geometry

( $x)$ Material properties appropriate and reasonable

( $x$ ) Mathematical derivations checked including dimensional consistency

$(x)$ Hand calculations checked for errors

( $x$ ) Assumptions explicitly stated and justified

( $x$ ) Computer software appropriate for task and used within range of validity

$(\cdot x)$ Use of resource outside range of established validity is justified

( $x$ ) Software runstreams correct and consistent with results

(x) Software output consistent with input

( $x$ ) Results consistent with applicable previous experimental or analytical findings

( $x$ ) Results and conclusions address all points and are consistent with task requirements and/or established limits or criteria

$(x)$ Conclusions consistent with analytical results and established limits

(NA) Uncertainty assessment appropriate and reasonable

(NA) other (define)

III. Comments:

ISOSHLD Code has been effectively applied to this project to meet the tight schedule.

IV. REVIEWER:J,S. Lan D.S. DATE: $3 / 24 / 97$ 


\section{DISTRIBUTION SHEET}

\begin{tabular}{|c|c|c|c|c|c|}
\hline \multirow{2}{*}{$\begin{array}{l}\text { To } \\
\text { Distribution }\end{array}$} & \multirow{2}{*}{\multicolumn{3}{|c|}{$\begin{array}{l}\text { From } \\
\text { Criticality and Shielding }\end{array}$}} & \multicolumn{2}{|l|}{ Page 1 of 1} \\
\hline & & & & \multicolumn{2}{|c|}{ Date March 24, 1997} \\
\hline \multicolumn{4}{|l|}{ Project Title/Work Order } & \multicolumn{2}{|c|}{ EDT No. 620300} \\
\hline \multicolumn{4}{|c|}{$\begin{array}{l}\text { Shielding Analys is for } 300 \text { Area Light Water Reactor Spent } \\
\text { Nuclear Fuel Within a Modified Multi-Canister Overpack Canister } \\
\text { in a Modified Multi-Canister Overpack Transportation Cask }\end{array}$} & \multicolumn{2}{|l|}{ ECN No. NA } \\
\hline Name & MSIN & $\begin{array}{l}\text { Text } \\
\text { With All } \\
\text { Attach. }\end{array}$ & Text Only & $\begin{array}{l}\text { Attach./ } \\
\text { Appendix } \\
\text { Only }\end{array}$ & $\begin{array}{l}\text { EDT/ECN } \\
\text { Only }\end{array}$ \\
\hline
\end{tabular}

A. B. Cartson

R3-86 X

S. R. Gedeon

HO-35 X

J. Greenborg

HO-35 X

J. S. Lan

HO-35 $X$

Central Files $(1+$ origina 1$)$

A3-88 $X$

